\title{
Systematic review and meta-analysis of the effects of high protein oral nutritional supplements on strength
}

\author{
A. L. Cawood ${ }^{1}$, R. J. Stratton ${ }^{1,2}$ and M. Elia ${ }^{2}$ \\ ${ }^{1}$ Medical Affairs, Nutricia Ltd, Wiltshire, BA14 OXQ and ${ }^{2}$ Institute of Human Nutrition, University of Southampton, \\ SO16 6YD, UK
}

Systematically obtained evidence on the effects of high protein oral nutritional supplements (ONS) in clinical practice is lacking. We have previously reported that ONS high in protein can significantly improve nutritional intake, weight and muscle tissue compared to control ${ }^{(1)}$. However it is not known if this is associated with improved functional outcomes. This systematic review and meta-analysis aimed to synthesise the evidence for high protein ONS ( $>20 \%$ energy from protein) ${ }^{(2)}$ versus control (routine care, placebo) on the functional outcome, strength.

Searches of electronic databases and bibliographies (up to Jan 2010) identified 36 randomised controlled trials (n 3790) in which multinutrient high protein ONS, were added to the diets of a range of patient groups including elderly, respiratory disease, hip fracture, cancer, GI disease and other critical and acute illnesses. Six RCT (n 3657) (prescribed daily intake 200-900 kcal; 15-60 g protein; intervention period 49days - 180days, mean 108days) reported skeletal muscle (handgrip) strength. Populations included elderly with a range of conditions (1RCT), respiratory (2RCT), hip fracture (2RCT) and GI disease (1RCT). Intervention was based mainly in the community (5RCT), with one trial starting in the hospital and continuing in the community setting. Meta-analysis was performed using Comprehensive Meta-Analysis v2.

In the six studies that reported handgrip strength one did not report any data but stated there were no differences. For the other five studies (n 275), mean changes in handgrip strength were greater in the ONS group (mean change in handgrip strength $+1.8 \mathrm{~kg}$ ) than the control group (mean change in handgrip strength $-0.08 \mathrm{~kg}$ ). In four of the five studies hand grip strength decreased in the control group. A meta-analysis, which could be undertaken on four of the five studies, showed significant effects in favour of the ONS group (1.76 kg (95\% CI 0.355-3170), n 240, p = 0.014, random effects model).

This systematic review and meta-analysis suggests that high protein ONS can significantly improve skeletal muscle strength (handgrip) compared to control.

1. Cawood AL et al. (2010) Clin Nutr Supps 5(2); 123-124.

2. Lochs H et al. (2006) Clin Nutr 25(2), 180-186. 